\title{
Determination of brain death under extracorporeal life support
}

\author{
Thomas Bein $^{1 *}\left(\mathbb{D}\right.$, Thomas Müller ${ }^{2}$ and Giuseppe Citerio $^{3}$
}

(0) 2019 Springer-Verlag GmbH Germany, part of Springer Nature

\begin{abstract}
Introduction
The worldwide use of extracorporeal lung/life support is increasing in severe lung failure (veno-venous extracorporeal membrane oxygenation, vvECMO) or in cardiac arrest/severe circulatory failure (veno-arterial ECMO, vaECMO). ECMO presents a new level of technology; but at the same time, ECMO has created a new field in medical ethics [1]. In recent years, the in-hospital mortality of patients treated with ECMO has remained high [2], and in specific indications for ECMO, such as vaECMO for cardiopulmonary resuscitation, the prognosis of (good) neurologic recovery is often unclear or poor. Consequently, the number of patients diagnosed with brain death under ECMO treatment will rise. Two retrospective analyses reported 161 brain-dead ECMO-supported patients (National French Registry of 22,270 donors after brain death between 2007 and 2013 [3]), and 100 brain deaths out of 356 ECMO patients with neurologic complications (Extracorporeal Life Support Organization data bank from 350 ECMO centers during 1992-2015: 4988 adults [4]), respectively. Since further increase of ECMO use is expected, and the estimation of brain death sometimes marks the end of strenuous therapeutic efforts, accurate knowledge about the specific challenges of brain death diagnosis under the circumstances of ECMO treatment is indispensable.
\end{abstract}

\section{Brain death diagnosis}

Although there is a general consensus on the concept of brain death in Europe, the concrete procedure to estimate brain death varies across the European countries

\footnotetext{
*Correspondence: Thomas.bein@ukr.de

${ }^{1}$ Department of Anesthesia and Operative Intensive Care, University Hospital Regensburg, Regensburg 93042, Germany

Full author information is available at the end of the article
}

[5]. The clinically assessed symptoms of unconsciousness, absence of brain stem reflexes, and apnea are the preconditions for further diagnostic steps. The modes, amounts, and timing of confirmatory technical or clinical tests are pronouncedly varied between countries-in Europe and worldwide. In general, the following steps are prescribed in most countries:

- Clinical examination of the complete termination of brain function (coma, failure of brain stem reflexes, apnea test).

- Proof of irreversibility:

- Repetition of clinical diagnosis and duration of coma after a certain time.

- Technical confirmatory tests.

\section{Specific aspects of brain death diagnosis in ECMO patients}

With the diagnosis of brain death, a careful protocol is required, which is adapted to nation-specific prescriptions. To pass the necessary steps successfully, the following distinctive aspects of care are required:

\section{Clinical examination}

Hemodynamic stability (systolic arterial pressure $>90 \mathrm{mmHg})$, adequate core temperature $\left(>36^{\circ} \mathrm{C}\right)$ and the absence of circulating analgesic, sedative, muscular blocking agents, or of severe electrolyte or glucose disorders are required for the correct assessment of brain death symptoms. In ECMO patients, specifically in vaE$\mathrm{CMO}$, a hemodynamic homeostasis may be hard to maintain. In a presentation of three adult patients with brain death determination under ECMO [6], no hemodynamic disturbances were reported during the complete procedure, while Giani et al. [7] demonstrated a 
moderate decrease in mean arterial pressure (median $61 \mathrm{mmHg}$ [49-75]) compared to non-ECMO patients (72 [63-93], $p<0.001)$, especially at the end of the apnea test.

\section{Apnea test}

While the documentation of apnea with the help of a specific test procedure is required in nearly all countries, considerable variations can be found in their individual laws. In most countries, a target hypercapnia level-documented via arterial $\mathrm{PaCO}_{2} \geq 60 \mathrm{mmHg}$-is prescribed; moreover, the regulations in some other countries (like Germany) demand a documented normocapnia before commencing the apnea test $\left(\mathrm{PaCO}_{2} 35-45 \mathrm{mmHg}\right)$. In ECMO patients, the $\mathrm{PaCO}_{2}$ level is mainly determined by the amount of extracorporeal $\mathrm{CO}_{2}$ removal, which is predominantly controlled by the sweep gas flow. Consequently, to perform the apnea test correctly, the sweep gas flow is reduced to approximately $1 \mathrm{l} / \mathrm{min}$. Simultaneously, the fraction of inspired oxygen to the membrane lung should be upregulated to $100 \%$ (Fig. 1). Apnea is visually verified by the lack of breathing after liberation from the ventilator. In patients suffering from severe lung failure (ARDS), the removal from mechanical ventilation may result in hypoxia. In order to avoid derecruitment and hypoxia during or after the apnea test, the application of positive end-expiratory pressure (PEEP via AMBU bag with PEEP valve) is recommended during an apnea test and a recruitment maneuver after the apnea test [7]. Although the performance of apnea testing-whether in ECMO or non-ECMO patients-is a challenging and potentially risky procedure, a retrospective analysis of 169 patients (25 on vaECMO) [7], despite a hypoxic episode $\left(\mathrm{PaO}_{2}<40 \mathrm{mmHg}\right.$ at the end of the apnea test), reported no further substantial complications and no procedure had to be aborted. As mentioned above, a moderate disturbance of hemodynamics was noticed in ECMO patients. These findings are confirmed by other case reports [8-10].

\section{Confirmatory technical tests}

The realization of confirmatory technical tests is facultative in some countries, and even mandatory in others [11]. The application of electroencephalography (EEG) or transcranial Doppler (TCD) sonography should be trouble-free in ECMO patients, while for angio-computer tomography (angio-CT) or cerebral scintigraphy,

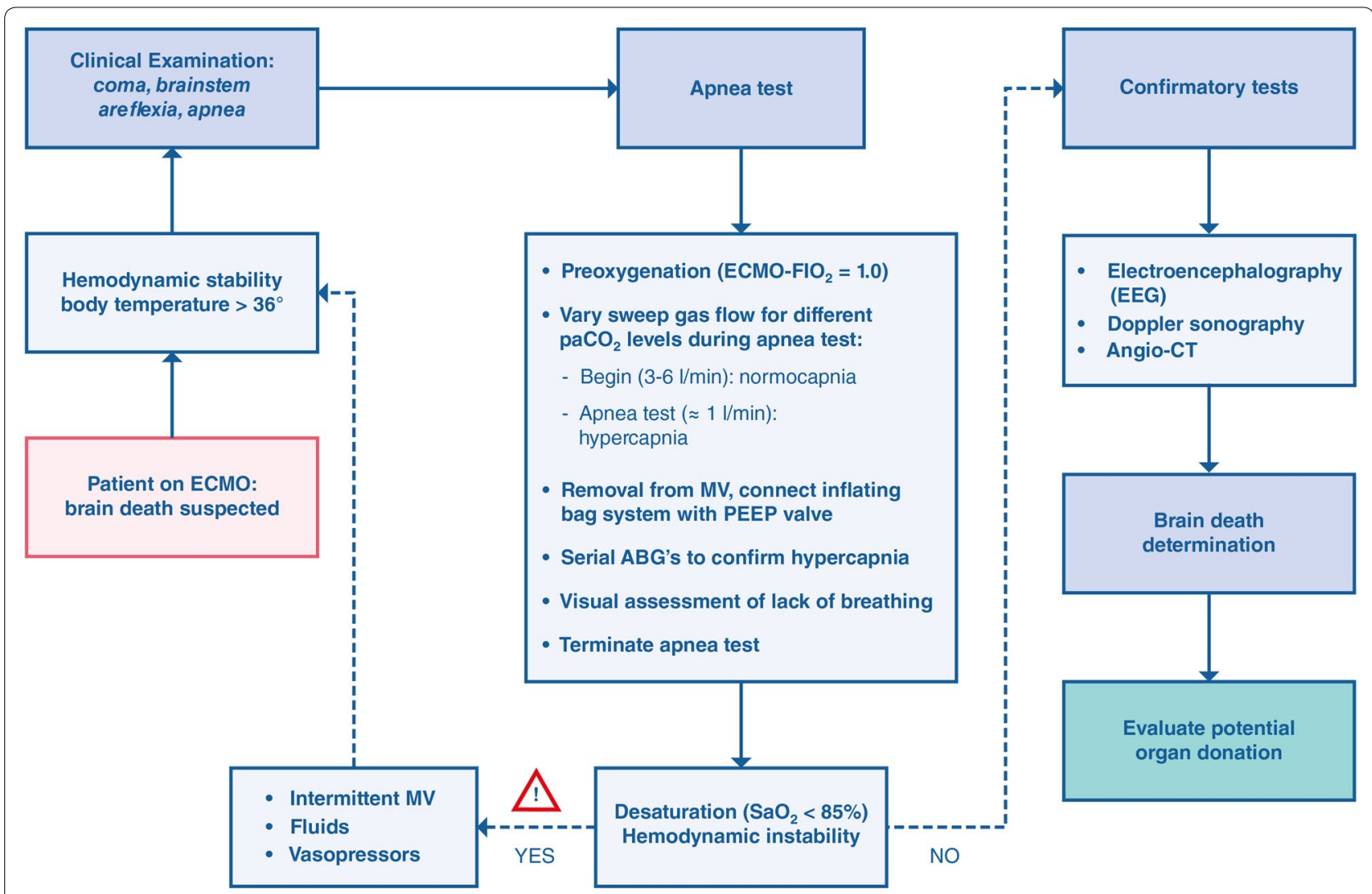

Fig. 1 Algorithm of the process of brain death estimation in patients on ECMO.MV mechanical Ventilation, ABG arterial blood gas, PEEP positive endexpiratory pressure 
the transportation of the ECMO patient is needed, which might be associated with hemodynamic or pulmonary instability and ECMO could affect the correct interpretation of the findings of these imaging techniques. In a recent investigation [12], TCD patterns seemed to be a reliable instrumental test for cerebral circulatory arrest in vaECMO patients only when a pulsatile cardiac flow was still maintained. Timing of contrast medium during angio-CT may be difficult for the same reasons. If possible, less invasive procedures for confirmatory tests (EEG, TCD sonography) should be preferred in order to allow maximal organ preservation.

\section{Organ donation in brain-dead ECMO patients}

As per the National French Registry report [3], 64 out of 161 ECMO patients (39.8\%) were organ donors. In a single center, cohort observation of 112 vaECMO patients after cardiac arrest [13], 25 patients suffered from brain death and $56 \%$ of these donated 39 organs with a $90 \%$ rate of good early function after transplantation. In a recent retrospective report from China [14], ECMO was used to provide support in unstable brain-dead donors on high doses of vasopressors resulting in a higher rate of good post-transplant liver function compared to non-stable donors without ECMO.

In conclusion, brain death determination in ECMO patients is expected to become an increasing occurrence. Its successful and legally conforming realization requires a correct and strict protocol, accurate knowledge among the intensive care staff, and the awareness of possible complications. In particular, the procedure of apnea testing might be associated with hemodynamic or respiratory alterations. Under the terms of careful evaluation and professional treatment, brain-dead ECMO patients are suitable for organ donation.

\footnotetext{
Author details

1 Department of Anesthesia and Operative Intensive Care, University Hospital Regensburg, Regensburg 93042, Germany. ${ }^{2}$ Department of Internal Medicine II, University Hospital Regensburg, Regensburg, Germany. ${ }^{3}$ School of Medicine and Surgery, Milano Bicocca University, Milan, Italy.
}

Compliance with ethical standards

Conflicts of interest

The authors declare no conflicts of interest.

\section{Publisher's Note}

Springer Nature remains neutral with regard to jurisdictional claims in published maps and institutional affiliations.

Received: 5 November 2018 Accepted: 19 December 2018

Published online: 9 January 2019

\section{References}

1. Bein T, Brodie D (2017) Understanding ethical decisions for patients on extracorporeal life support. Intensive Care Med 43:1510-1511

2. Karagiannidis C, Brodie D, Strassmann S, Stoelben E, Philipp A, Bein T, Müller T, Windisch W (2016) Extracorporeal membrane oxygenation: evolving epidemiology and mortality. Intensive Care Med 42:889-896

3. Bronchard R, Durand L, Legeai C, Cohen J, Guerrini P, Bastien O (2017) Brain-dead donors on extracorporeal membrane oxygenation. Crit Care Med 45:1734-1741

4. Lorusso R, Gelsomino S, Parise O, Di Mauro M, Barili F, Geskes G, Vizzardi E, Rycus PT, Muellenbach R, Mueller T, Pesenti A, Combes A, Peek G, Frenckner B, Di Nardo M, Swol J, Maessen J, Thiagarajan RR (2017) Neurologic injury in adults supported with veno-venous extracorporeal membrane oxygenation for respiratory failure: findings from the Extracorporeal Life Support Organization database. Crit Care Med 45:1389-1397

5. Citerio G, Murphy PG (2015) Brain death: the European perspective. Semin Neurol 35:139-144

6. Muralidharan R, Mateen FJ, Shinohara RT, Schears GJ, Wijdicks EF (2011) The challenges with brain death determination in adult patients on extracorporeal membrane oxygenation. Neurocrit Care 14:423-426

7. Giani M, Scaravilli V, Colombo SM, Confalonieri A, Leo R, Maggioni E, Avalli L, Vargiolu A, Citerio G (2016) Apnea test during brain death assessment in mechanically ventilated and ECMO patients. Intensive Care Med 42:72-81

8. Goswami S, Evans A, Das B, Prager K, Sladen RN, Wagener G (2013) Determination of brain death by apnea test adapted to extracorporeal cardiopulmonary resuscitation. J Cardiothorac Vasc Anesth 27:312-314

9. Migliaccio ML, Zagli G, Cianchi G, Lazzeri C, Bonizzoli M, Cecchi A, Anichini V, Gensini GF, Peris A (2013) Extracorporeal membrane oxygenation in brain-death organ and tissues donors: a single-centre experience. $\mathrm{Br}$ J Anaesth 111:673-674

10. Shah V, Lazaridis C (2015) Apnea testing on extracorporeal membrane oxygenation: case report and literature review. J Crit Care 30:784-786

11. Haupt WF, Rudolf J (1999) European brain death codes: a comparison of national guidelines. J Neurol 246:432-437

12. Marinoni M, Cianchi G, Trapani S, Migliaccio ML, Bonizzoli M, Gucci L, Cramaro A, Gallerini A, Picciafuochi F, Valente S, Peris A (2018) Retrospective analysis of transcranial Doppler patterns in veno-arterial extracorporeal membrane oxygenation patients: feasibility of cerebral circulatory arrest diagnosis. ASAIO J 64:175-182

13. Casadio MC, Coppo A, Vargiolu A, Villa J, Rota M, Avalli L, Citerio G (2017) Organ donation in cardiac arrest patients treated with extracorporeal CPR: a single centre observational study. Resuscitation 118:133-139

14. Fan $X$, Chen Z, Nasralla D, Zeng X, Yang J, Ye S, Zhang Y, Peng G, Wang Y, Ye Q (2016) The organ preservation and enhancement of donation success ratio effect of extracorporeal membrane oxygenation in circulatory unstable brain death donor. Clin Transpl 30:1306-1313 